\title{
| A complexidade da multimorbidade
}

\author{
| The complexity of multimorbidity
}

\author{
Sandro Rodrigues Batista \\ Faculdade de Medicina. Universidade Federal de Goiás. \\ Diretor de Pesquisa da Sociedade Brasileira de Medicina de Família.
}

\begin{abstract}
A multimorbidade, ou seja, a presença de duas ou mais morbidades em um mesmo indivíduo, foi descrita pela primeira vez na Alemanha em 1976, mas só a partir dos anos 90 passou a despertar maior interesse na comunidade científica. Estimativas em relação à prevalência de multimorbidade variam muito (13 a 66\%) e tem colocado essa condição como um dos principais desafios para os sistemas de saúde. A multimorbidade não se apresenta como uma exclusividade de pacientes de faixas etárias mais avançadas apesar desse estrato ter maior acometimento, está associada com maior mortalidade, capacidade funcional reduzida, aumento da demanda por cuidados em saúde e maiores custos.

Os pacientes com múltiplas morbidades apresentam desafios únicos para os profissionais de saúde e causam nos mesmos dois tipos básicos de reação: a motivação pela complexidade e o desespero pelo mesmo motivo. Esse nível de complexidade característico na abordagem da pessoa com multimorbidade faz como que cada situação seja única, a natureza do seu sistema. Evidências e instrumentos para esse manejo são escassos, o que torna o desespero citado anteriormente quase uma realidade.

Protocolos e diretrizes são, em sua grande maioria, desenhados para condições isoladas. Esses estão mais na dimensão do "OU" (pacientes com HAS ou DM ou DPOC) sendo que na prática clínica é exigida com frequência a dimensão do "E" (pacientes com HAS e DM e DPOC). Deforma complementar, os estudos de intervenção são desenhados para testar um tratamento único e ainda excluem de seus grupos de estudos pacientes que apresentam multimorbidade. Fatores socioeconômicos apresentam nesse contexto um papel fundamental havendo evidências de maior prevalência de multimorbidade nos estratos sociais com maior privação. Quando uma dessas morbidades é do campo da saúde mental essa iniquidade torna-se ainda mais evidente. Nesse contexto fica a clássica pergunta: e as situações únicas de cada paciente? Como manejá-las?

Outra fundamental dimensão de avaliação desse contexto é a importância extrema que se tem conferido ao número de morbidades em um paciente. A carga de morbidade, ou seja, o impacto total de diferentes morbidades num indivíduo tem muito mais importância para o cuidado da saúde deste. Ao analisarmos o número de prováveis interações medicamentosas às quais esses pacientes estão expostos, há maior relevância clínica e para o cuidado a forma como essas morbidades se inter-relacionam e impactam no paciente do que simplesmente o número existente das mesmas.

Nesse cenário, a atenção primária à saúde (APS) tem-se configurado como uma das estratégias mais promissoras para o manejo do cuidado desses pacientes. É fundamental para esses pacientes a execução da coordenação do cuidado. Esse atributo essencial da APS advoga que os profissionais dessas equipes de saúde são responsáveis por harmonizar as diversas intervenções que podem ocorrer no processo do cuidar. Ademais, questões como a
\end{abstract}


longitudinalidade, o vínculo e o método clínico centrado na pessoa contribuem sobremaneira para a condução dessas situações. Além disso, por esses pacientes estarem mais vulneráveis à iatrogenia (entre elas a cascata de prescrições) faz-se necessário a execução da prevenção quaternária, e assim, proteger nosso paciente de intervenções desnecessárias e eticamente inaceitáveis.

Pacientes com multimorbidade configuram-se mais como regra do que como exceção na rotina dos serviços de APS. Necessitamos urgentemente de repensar essa prática e instrumentalizar seus profissionais com ferramentas para o seu adequado manejo para que esses possam efetivar a coordenação do cuidado. De forma complementar, também preparar os demais níveis de atenção do sistema de saúde para que façam as intervenções necessárias, quando acionados é importante. Por fim, fica claro que precisamos organizar sistema de saúde para o manejo de pacientes com multimorbidade e empoderar cada nível de atenção à saúde conforme sua potencialidade e efetividade nesse cuidado. Afinal, esses pacientes são complexos ou os sistemas de saúde são simples? 